\title{
Exponential and polynomial stabilization of the Kirchhoff string by nonlinear boundary control
}

\author{
Yuhu $\mathrm{Wu}^{1 *}$ and Jianmin Wang ${ }^{2}$
}

\section{"Correspondence:}

wuyuhu51@gmail.com

'Department of Mathematics,

Harbin University of Science and

Technology, Harbin, 150080,

P.R. China

Full list of author information is

available at the end of the article

\begin{abstract}
This paper addresses the stabilization problem of the nonlinear Kirchhoff string using nonlinear boundary control. Nonlinear boundary control is the negative feedback of the transverse velocity of the string at one end, which satisfies a polynomial-type constraint. Employing the multiplier method, we establish explicit exponential and polynomial stability for the Kirchhoff string. The theoretical results are assured by numerical results of the asymptotic behavior for the system.
\end{abstract}

\section{Introduction}

Stabilization and vibration controllability of string or beam systems arising from different engineering backgrounds has attracted attention of many researchers [1-4]. In particular, boundary feedback stabilization of string and beam systems has become an important research area [5-7]. This is because, in a practice system, vibration is more easily controlled through a boundary point than using point sensors or actuators away from the boundaries $[8,9]$.

There are several nonlinear mathematical models that describe the transversal vibration of stretched strings. One such model is presented in the following equation:

$$
y_{t t}(x, t)=\left[a+b \int_{0}^{1} y_{x}^{2}(x, t) d x\right] y_{x x}(x, t)
$$

for all $x \in(0,1)$ and $t \geq 0$, where $a>0, b \geq 0$ are two constants. Obviously, the above equation is a simple prototype of the classical equation

$$
\rho h y_{t t}(x, t)=\left[T_{0}+\frac{h E}{2 l} \int_{0}^{l} y_{x}^{2}(x, t) d x\right] y_{x x}(x, t)
$$

which was proposed by Kirchhoff [10]. Here $l$ is the length of the string; $E$ is Young's modulus of the material; $\rho$ is density; $h$ is the area of the cross section; $y(x, t)$ is the transversal displacement of the point $x$ of the string at time $t$. This model has been studied by researchers from the physical and mathematical points of view; see, e.g., references [11-13] and the references therein.

(c) 2013 Wu and Wang; licensee Springer. This is an Open Access article distributed under the terms of the Creative Commons Attribution License (http://creativecommons.org/licenses/by/2.0), which permits unrestricted use, distribution, and reproduction in any medium, provided the original work is properly cited. 


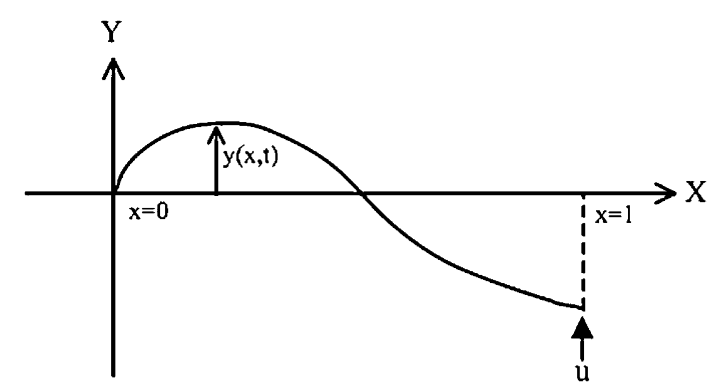

Figure 1 Schematic of the nonlinear Kirchhoff string with boundary control.

In this paper, we consider Kirchhoff string (1) with the following boundary conditions (see Figure 1):

$$
\begin{aligned}
& y(0, t)=0, \\
& T(t) y_{x}(1, t)=u(t)
\end{aligned}
$$

for all $t \geq 0 . T(t)$ denotes the tension in the string at time $t$. The boundary condition in equation (2) implies that the string is fixed at $x=0$. The boundary condition in equation (3) represents the balance of the transversal component of the tension in the string and the control input $u$ which is applied transversally at $x=1$. Because the tension in the string represented by equation (1) is not constant and is given by

$$
T(t)=a+b \int_{0}^{1} y_{x}^{2}(x, t) d x
$$

for all $t \geq 0$ (see [14]), the boundary condition in equation (3) can be written as

$$
\left[a+b \int_{0}^{1} y_{x}^{2}(x, t) d x\right] y_{x}(1, t)=u(t)
$$

Shahruz and Krishna [13] investigated the stabilization of Kirchhoff string (1) with a linear negative velocity control, which means the boundary control $u$ has a linear negative velocity feedback form

$$
u(t):=u\left(y_{t}(1, t)\right)=-L y_{t}(1, t)
$$

for all $t \geq 0$, where $L$ is a positive constant. They established exponential stability. In [15], the absolute stability of the Kirchhoff string (1) with linear sector boundary control was considered. It is well known that linear strings represented by equation (1), for which $b=0$, can be stabilized by the control law in equation (6); see e.g., references [16-18]. Moreover, Shahruz [19], Fung et al. [5], and Li and Hou [20] developed linear boundary control laws for axially moving strings. It is worth mentioning that Kobayashi [21] designed a linear parallel compensator based on boundary displacement observer and proved the string (1) can be stabilized by parallel compensator control.

In the literature mentioned above, such as $[13,16]$ and $[20]$, the exponential stabilization result for various string systems by linear boundary control mainly relies on the Lyapunov 
direct method. In this work, we investigate the stabilization of string (1) with a more general and 'flexible' boundary control (see hypothesis $(\mathrm{H})$ in Section 2). The feedback function $u$ is not required to satisfy a strict control law such as (6), but just satisfies some appropriate polynomial-type constraint. In this general boundary control case, it seems that the Lyapunov direct method is no more applicable. So, we need to use a more meticulous method to deal with the stabilization problem. Applying the multiplier method, we establish not only exponential stability result but also polynomial stability result for Kirchhoff string (1).

The remainder of this technical paper is arranged as follows. Section 2 describes the model of the Kirchhoff nonlinear string and introduces the control assumption. The problem of exponential and polynomial stability is addressed in Section 3. Finally, a numerical example is demonstrated where the nonlinear distributed parameter infinite-dimensional equation is solved by applying the finite element method in Section 4.

\section{Problem formulation}

Consider the nonlinear Kirchhoff string model as shown in Figure 1. For the sake of easy reading and later referring, the governing equation, the boundary conditions and the initial functions are put together as

$$
\left\{\begin{array}{l}
y_{t t}(x, t)=\left[a+b \int_{0}^{1} y_{x}^{2}(x, t) d x\right] y_{x x}(x, t), \\
y(0, t)=0 \\
{\left[a+b \int_{0}^{1} y_{x}^{2}(x, t) d x\right] y_{x}(1, t)=u\left(y_{t}(1, t)\right)} \\
y(x, 0)=f(x), \quad y_{t}(x, 0)=g(x)
\end{array}\right.
$$

for all $x \in(0,1)$ and $t \geq 0$. Here $f(x)$ and $g(x)$ in equation (7d) are the initial displacement and velocity of the string, respectively. We assume that $f \in C^{1}[0,1], g \in C[0,1]$ and that least one of the functions $f$ or $g$ is not identically zero over $[0,1]$.

To obtain a precise stabilization result, we make the following hypothesis on the continuous control feedback $u: R \rightarrow R$ :

(H) There exist constants $L_{2} \geq L_{1}>0$ and $r \geq 1$ such that

$$
u(w) \in \bar{\Omega}(w):= \begin{cases}-\operatorname{Co}\left\{L_{1} w, L_{2} w\right\}, & |w|>1, \\ -\operatorname{Co}\left\{L_{1} w^{r}, L_{2} w^{\frac{1}{r}}\right\}, & |w| \leq 1\end{cases}
$$

Remark 2.1 Obviously, condition (H) is equivalent to, for all $w \in R$,

$$
\left\{\begin{array}{l}
u(w) w \leq 0, \\
L_{1} \min \left\{|w|,|w|^{r}\right\} \leq|u(w)| \leq L_{2} \max \left\{|w|,|w|^{\frac{1}{r}}\right\} .
\end{array}\right.
$$

Obviously, hypothesis $(\mathrm{H})$ is a 'flexible' and 'robust' condition, which allows the feedback function $u$ to vary in an appropriate geometric region given by a polynomial-type constraint. For example, Figure 2 illustrates a feedback control $u$ satisfying a linear sector constraint $(\mathrm{H})$ with $r=1, L_{1}=1, L_{2}=2$, and Figure 3 shows a feedback control $u$ satisfying a nonlinear constraint $(\mathrm{H})$ with $r=2, L_{1}=1, L_{2}=2$. Since $u\left(y_{t}(1, t)\right) y_{t}(1, t) \leq 0$, for all $t \geq 0$, the boundary control (7c) is the negative feedback of transversal velocity of the string at $x=1$. 


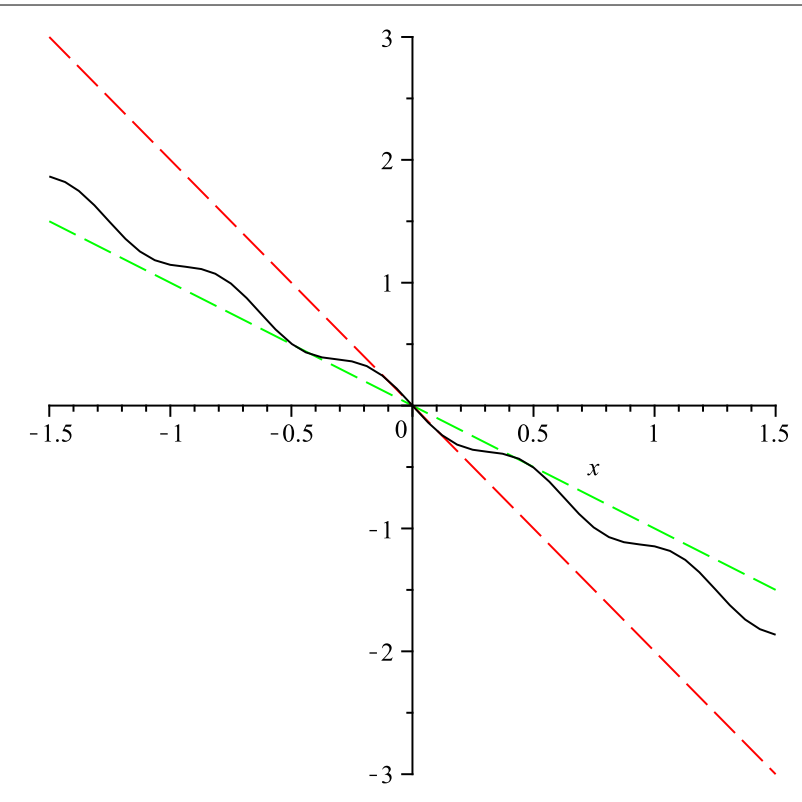

Figure 2 Linear sector constraint.

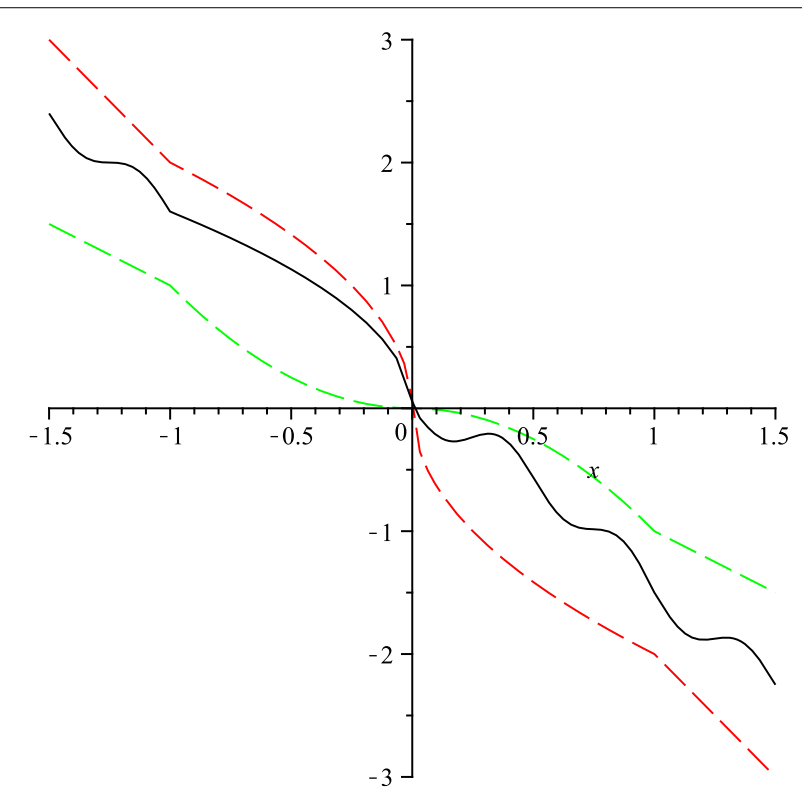

Figure 3 Nonlinear sector constraint.

For the existence and uniqueness of the solution of the general Kirchhoff equation, we refer to $[11,12]$ and references therein. In this work, we study the stabilization of the string in (7a) by this negative feedback boundary control $u$, which provides a dissipative effect.

Remark 2.2 According to boundary condition (7b) at $x=0$, we easily get

$$
y_{t}(0, t)=0 \quad \text { for all } t \geq 0 \text {. }
$$


We define the natural energy function of time for system (7a)-(7d) and denote it by $t \rightarrow$ $E(t)$. The scalar-valued function $E$ is defined as

$$
E(t):=\frac{1}{2} \int_{0}^{1} y_{t}^{2}(x, t) d x+\frac{1}{2} \int_{0}^{1} a y_{x}^{2}(x, t) d x+\frac{b}{4}\left[\int_{0}^{1} y_{x}^{2}(x, t) d x\right]^{2} .
$$

Especially, from the initial displacement and velocity condition (7d), we obtain the initial energy as

$$
E(0)=\frac{1}{2} \int_{0}^{1}\left[g^{2}(x)+a f_{x}^{2}(x)\right] d x+\frac{b}{4}\left[\int_{0}^{1} f_{x}^{2}(x) d x\right]^{2} .
$$

Since at least one of the functions $f$ and $g$ is not identically zero over $(0,1)$ we have $E(0)>0$.

\section{Stabilization by boundary control}

In this section we state and prove our main result. For this purpose we establish several lemmas.

Lemma 3.1 Let $y(\cdot, \cdot)$ be the solution for system (7a)-(7d). Then

$$
\begin{aligned}
& \int_{0}^{1} y_{x} y_{x t} d x=y_{x}(1, t) y_{t}(1, t)-\int_{0}^{1} y_{x x} y_{t} d x \\
& \int_{0}^{1} x y_{x} y_{x x} d x=\frac{1}{2} y_{x}^{2}(1, t)-\frac{1}{2} \int_{0}^{1} y_{x}^{2}(x, t) d x \\
& \int_{0}^{1} x y_{x} y_{t t} d x=\int_{0}^{1}\left(\left[x y_{x} y_{t}\right]_{t}+\frac{1}{2} y_{t}^{2}\right) d x-\frac{1}{2} y_{t}^{2}(1, t) .
\end{aligned}
$$

Proof See the Appendix.

Now, we give a property of the energy function $E$.

Proposition 3.1 The time-derivative of the energy function $E$ in equation (10), along the solution of system (7a)-(7d) satisfies

$$
E^{\prime}(t)=u(t) y_{t}(1, t) \leq 0
$$

for all $t \geq 0$.

Proof Differentiating the energy function (10) with respect to $t$, we get

$$
E^{\prime}(t)=\int_{0}^{1} y_{t} y_{t t} d x+\left[a+b \int_{0}^{1} y_{x}^{2} d x\right] \int_{0}^{1} y_{x} y_{x t} d x
$$

According to equation (11) and boundary control (7c), we get

$$
\begin{aligned}
& {\left[a+b \int_{0}^{1} y_{x}^{2} d x\right] \int_{0}^{1} y_{x} y_{x t} d x} \\
& \quad=u(t) y_{t}(1, t)-\left[a+b \int_{0}^{1} y_{x}^{2} d x\right] \int_{0}^{1} y_{x x} y_{t} d x
\end{aligned}
$$


Substituting equation (16) into equation (15) and observing (7a), we obtain

$$
\begin{aligned}
E^{\prime}(t) & =u(t) y_{t}(1, t)+\int_{0}^{1} y_{t t} y_{t} d x-\left[a+b \int_{0}^{1} y_{x}^{2} d x\right] \int_{0}^{1} y_{x x} y_{t} d x \\
& =u(t) y_{t}(1, t)
\end{aligned}
$$

for all $t \geq 0$. We obtain equation (14).

Remark 3.1 From Proposition 3.1, we obtain the energy identity for system (7a)-(7d),

$$
E(S)-E(T)=\int_{T}^{S} u(t) y_{t}(1, t) d t .
$$

Therefore, the energy $E$ is a decreasing function of time.

During the subsequent stability analysis, we utilize the following inequality.

Lemma 3.2 Let $y(\cdot, \cdot)$ be the solution for system (7a)-(7d). Then

$$
\int_{0}^{1} x y_{x}(x, t) y_{t}(x, t) d x \leq \max \left\{1, \frac{1}{a}\right\} E(t)
$$

for all $t \geq 0$.

Proof Applying the Cauchy-Schwarz inequality, we get

$$
\begin{aligned}
\int_{0}^{1} x y_{x}(x, t) y_{t}(x, t) d x & \leq \frac{1}{2} \int_{0}^{1} x^{2} y_{x}^{2} d x+\frac{1}{2} \int_{0}^{1} y_{t}^{2} d x \\
& \leq \frac{1}{2} \int_{0}^{1} y_{x}^{2} d x+\frac{1}{2} \int_{0}^{1} y_{t}^{2} d x
\end{aligned}
$$

for all $t \geq 0$. On the other hand, the definition of energy function (10) implies

$$
\frac{a}{2} \int_{0}^{1} y_{x}^{2} d x+\frac{1}{2} \int_{0}^{1} y_{t}^{2} d x \leq E(t)
$$

for all $t \geq 0$. It follows from the above inequality that

$$
\frac{1}{2} \int_{0}^{1} y_{x}^{2} d x+\frac{1}{2} \int_{0}^{1} y_{t}^{2} d x \leq \begin{cases}E(t), & a \geq 1, \\ \frac{1}{a} E(t), & 0<a<1 .\end{cases}
$$

Together with (19) and (20), we get equation (18). Hence we complete the proof of Lemma 3.2.

Now, we present a Gronwall-type lemma (see Komornik [22], pp.124), which will play an essential role when establishing the stabilization result.

Lemma 3.3 Let $G: R_{+} \rightarrow R_{+}$be a non-increasing function. Assume that there exists a constant $\omega>0$ such that

$$
\int_{T}^{+\infty} G^{q+1}(t) d t \leq \frac{1}{\omega} G(T) \quad \text { for all } T \geq 0 .
$$


Then the following estimation is true, for all $t \geq 0$,

$$
\begin{cases}G(t) \leq G(0) e^{1-\omega t} & \text { if } q=0 \\ G(t) \leq G(0)\left(\frac{1+q}{q \omega t}\right)^{\frac{1}{q}} & \text { if } q>0\end{cases}
$$

We give a priori estimation for the energy function $E(t)$, which was established in [15]. For the sake of completeness, we give the proof here.

Lemma 3.4 The energy function $E$ in equation (10), along the solution of system (7a)-(7d), satisfies

$$
E(t) \leq-\int_{0}^{1}\left[x y_{x} y_{t}\right]_{t} d x+\frac{1}{2} y_{t}^{2}(1, t)+\frac{1}{2 a} u^{2}\left(y_{t}(1, t)\right)
$$

for all $t \geq 0$.

Proof We multiply equation (7a) by $x y_{x}(x, t)$ and do integration over $[0,1]$, with respect to $x$. We obtain

$$
\begin{aligned}
0= & \int_{0}^{1} x y_{x}\left(y_{t t}-\left[a+b \int_{0}^{1} y_{x}^{2} d x\right] y_{x x}\right) d x \\
= & \int_{0}^{1}\left(\left[x y_{x} y_{t}\right]_{t}+\frac{1}{2} y_{t}^{2}\right) d x-\frac{1}{2} y_{t}^{2}(1, t) \\
& -\frac{1}{2}\left[a+b \int_{0}^{1} y_{x}^{2} d x\right]\left(y_{x}^{2}(1, t)-\int_{0}^{1} y_{x}^{2} d x\right),
\end{aligned}
$$

using equations (13) and (12) in Lemma 3.1. It follows from (10) that

$$
\begin{aligned}
& \frac{1}{2} \int_{0}^{1} y_{t}^{2} d x+\frac{1}{2}\left[a+b \int_{0}^{1} y_{x}^{2} d x\right] \int_{0}^{1} y_{x}^{2} d x \\
& =E(t)+\frac{b}{4}\left[\int_{0}^{1} y_{x}^{2} d x\right]^{2} .
\end{aligned}
$$

Since $a+b \int_{0}^{1} y_{x}^{2} d x \geq a$, according to boundary control (7c), we have

$$
\left[a+b \int_{0}^{1} y_{x}^{2} d x\right] y_{x}^{2}(1, t) \leq \frac{1}{a} u^{2}\left(y_{t}(1, t)\right) .
$$

Hence, substituting equation (23) into equation (22) and using equation (24), one has

$$
\begin{aligned}
E(t) & +\frac{b}{4}\left[\int_{0}^{1} y_{x}^{2} d x\right]^{2} \\
& =-\int_{0}^{1}\left[x y_{x} y_{t}\right]_{t} d x+\frac{1}{2} y_{t}^{2}(1, t)+\frac{1}{2}\left[a+b \int_{0}^{1} y_{x}^{2} d x\right] y_{x}^{2}(1, t) \\
& \leq-\int_{0}^{1}\left[x y_{x} y_{t}\right]_{t} d x+\frac{1}{2} y_{t}^{2}(1, t)+\frac{1}{2 a} u^{2}\left(y_{t}(1, t)\right) .
\end{aligned}
$$

Since $\frac{b}{4}\left[\int_{0}^{1} y_{x}^{2} d x\right]^{2} \geq 0$, we complete the proof of Lemma 3.4. 
Lemma 3.5 For any constant $q \geq 0$, the energy function $E$ along the solution of system (7a)-(7d) satisfies the following estimation, for all $S>T \geq 0$,

$$
\begin{aligned}
\int_{T}^{S} E^{q+1}(t) d t \leq & C_{1} E^{q+1}(T)+\frac{1}{2} \int_{T}^{S} E^{q}(t) y_{t}^{2}(1, t) d t \\
& +\frac{1}{2 a} \int_{T}^{S} E^{q}(t) u^{2}\left(y_{t}(1, t)\right) d t
\end{aligned}
$$

where $C_{1}=\frac{3 q+2}{q+1} \max \left\{1, \frac{1}{a}\right\}$.

Proof According to inequality (21) in Lemma 3.4, we have, for all $0 \leq T<S$,

$$
\begin{aligned}
\int_{T}^{S} E^{q+1}(t) d t \leq & -\int_{T}^{S} E^{q}(t) \int_{0}^{1}\left[x y_{x} y_{t}\right]_{t} d x d t \\
& +\frac{1}{2} \int_{T}^{S} E^{q}(t) y_{t}^{2}(1, t) d t+\frac{1}{2 a} \int_{T}^{S} E^{q}(t) u^{2}\left(y_{t}(1, t)\right) d t .
\end{aligned}
$$

Moreover, using integration by parts, we get

$$
\begin{aligned}
\int_{T}^{S} E^{q}(t) \int_{0}^{1}\left[x y_{x} y_{t}\right]_{t} d x d t= & \int_{T}^{S}\left[E^{q}(t) \int_{0}^{1} x y_{x} y_{t} d x\right]_{t} d t \\
& -\int_{T}^{S} q E^{\prime}(t) E^{q-1}(t) \int_{0}^{1}\left(x y_{x} y_{t}\right) d x d t
\end{aligned}
$$

Hence, inequality (26) becomes

$$
\begin{aligned}
\int_{T}^{S} E^{q+1}(t) d t \leq & A_{1}+A_{2}+\frac{1}{2} \int_{T}^{S} E^{q}(t) y_{t}^{2}(1, t) d t \\
& +\frac{1}{2 a} \int_{T}^{S} E^{q}(t) u^{2}\left(y_{t}(1, t)\right) d t
\end{aligned}
$$

where

$$
\begin{aligned}
& A_{1}:=-\int_{T}^{S}\left[E^{q}(t) \int_{0}^{1} x y_{x} y_{t} d x\right]_{t} d t, \\
& A_{2}:=\int_{T}^{S} q E^{\prime}(t) E^{q-1}(t) \int_{0}^{1}\left(x y_{x} y_{t}\right) d x d t .
\end{aligned}
$$

Firstly, we estimate $A_{1}$ and $A_{2}$,

$$
\begin{aligned}
A_{1} & =-\int_{T}^{S}\left[E^{q}(t) \int_{0}^{1} x y_{x} y_{t} d x\right]_{t} d t \\
& \leq\left|E^{q}(T) \int_{0}^{1} x y_{x}(x, T) y_{t}(x, T) d x\right|+\left|E^{q}(S) \int_{0}^{1} x y_{x}(x, S) y_{t}(x, S) d x\right| \quad \text { by (18)) } \\
& \leq \max \left\{1, \frac{1}{a}\right\} E^{q+1}(T)+\max \left\{1, \frac{1}{a}\right\} E^{q+1}(S) \\
& \leq 2 \max \left\{1, \frac{1}{a}\right\} E^{q+1}(T)
\end{aligned}
$$


where the last inequality follows from the fact $E(t)$ is a decreasing function. On the other hand,

$$
\begin{aligned}
A_{2} & =\int_{T}^{S} q E^{\prime}(t) E^{q-1}(t) \int_{0}^{1} x y_{x} y_{t} d x d t \\
& \leq \int_{T}^{S}\left|q E^{\prime}(t) E^{q-1}(t)\right|\left|\int_{0}^{1} x y_{x} y_{t} d x\right| d t \\
& \leq \max \left\{1, \frac{1}{a}\right\}\left|\int_{T}^{S} q E^{\prime}(t) E^{q}(t) d t\right| \quad(\text { by }(18)) \\
& =\frac{q}{q+1} \max \left\{1, \frac{1}{a}\right\}\left|E^{q+1}(S)-E^{q+1}(T)\right| \\
& \leq \frac{q}{q+1} \max \left\{1, \frac{1}{a}\right\} E^{q+1}(T) .
\end{aligned}
$$

Finally, inserting the two inequalities, (28) and (29), in (27), we get inequality (25). This completes the proof of Lemma 3.5.

We now state the main stabilization result for system (7a)-(7d).

Theorem 3.1 Assume that assumption $(\mathrm{H})$ holds. Then there exist three constants $k_{1}, k_{2}, \sigma>0$ such that, for all $t \geq 0$,

$$
\begin{cases}E(t) \leq k_{1} e^{-\sigma t} & \text { if } r=1 \\ E(t) \leq k_{2} t^{-\frac{2}{r-1}} & \text { if } r>1\end{cases}
$$

Remark 3.2 It is worth to mention that Theorem 2.4 in [13] can be viewed as a special cases of Theorem 3.1. Indeed, in the linear control case (6), the exponential stability in Theorem 3.1 coincides with the result in [13].

Proof of Theorem 3.1 We distinguish two cases related to the parameter $r$ to establish the energy decay rate.

Case (I): $r=1$;

Case (II): $r>1$.

In Case (I), we choose $q=0$. According to hypothesis $(\mathrm{H})$, we know that

$$
s^{2} \leq-\frac{1}{L_{1}} u(s) s, \quad u^{2}(s) \leq-L_{2} u(s) s \quad \text { for all } s \in R
$$

Hence, from inequality (25) and equation (14), we deduce that, for all $S>T \geq 0$,

$$
\begin{aligned}
\int_{T}^{S} E(t) d t & \leq C_{1} E(T)+\frac{1}{2} \int_{T}^{S}\left[y_{t}^{2}(1, t)+\frac{1}{a} u^{2}\left(y_{t}(1, t)\right)\right] d t \\
& \leq C_{1} E(T)-\frac{a+L_{1} L_{2}}{2 a L_{1}} \int_{T}^{S} u(t) y_{t}(1, t) d t \\
& =C_{1} E(T)+\frac{a+L_{1} L_{2}}{2 a L_{1}} \int_{T}^{S}-E^{\prime}(t) d t \\
& \leq C_{2} E(T),
\end{aligned}
$$

where $C_{2}=C_{1}+\frac{1}{2}\left(\frac{1}{L_{1}}+\frac{L_{2}}{a}\right)$ and $C_{1}$ is given in Lemma 3.5. 
Now we deal with Case (II). In this case, we choose $q=\frac{r-1}{2}>0$. We first admit the following fact (the proof is given in the Appendix).

Claim 1 For any $\delta>0$, we have the following estimates, for all $S>T \geq 0$,

$$
\begin{aligned}
\int_{T}^{S} E^{q}(t) y_{t}^{2}(1, t) d t \leq & \frac{r-1}{r+1} \delta^{\frac{r+1}{r-1}} \int_{T}^{S} E^{q} \frac{r+1}{r-1}(t) d t \\
& +\frac{2 \delta^{-\frac{r+1}{2}}}{(r+1) L_{1}} E(T)+\frac{1}{(q+1) L_{1}} E^{q+1}(T), \\
\int_{T}^{S} E^{q}(t) u^{2}\left(y_{t}(1, t)\right) d t \leq & \frac{r-1}{r+1} \delta^{\frac{r+1}{r-1}} \int_{T}^{S} E^{q \frac{r+1}{r-1}}(t) d t \\
& +\frac{2 L_{2}^{r} \delta^{-\frac{r+1}{2}}}{(r+1)} E(T)+\frac{L_{2}}{(q+1)} E^{q+1}(T) .
\end{aligned}
$$

Now, inserting inequalities (32) and (33) into (25), we obtain, for all $S>T \geq 0$,

$$
\begin{aligned}
\int_{T}^{S} E^{q+1}(t) d t \leq & \left(\frac{1}{2}+\frac{1}{2 a}\right) \frac{r-1}{r+1} \delta^{\frac{r+1}{r-1}} \int_{T}^{S} E^{q^{\frac{r+1}{r-1}}}(t) d t \\
& +C_{3} E^{q+1}(T)+C_{4} E(T),
\end{aligned}
$$

where $C_{3}=C_{1}+\frac{a+L_{1} L_{2}}{2 a(q+1) L_{1}}, C_{4}=\frac{1}{(r+1) L_{1}} \delta^{-\frac{r+1}{2}}+\frac{L_{2}^{r}}{(r+1) a} \delta^{-\frac{r+1}{2}}$ and $C_{1}$ is given in Lemma 3.5. Now we choose $\delta=\left[\left(1+\frac{1}{a}\right) \frac{r-1}{r+1}\right]^{-\frac{r-1}{r+1}}$. Then it is obvious that $\left(1+\frac{1}{a}\right) \frac{r-1}{r+1} \delta^{\frac{r+1}{r-1}}=1$. Hence, inequality (34) becomes

$$
\int_{T}^{S} E^{q+1}(t) d t \leq \frac{1}{2} \int_{T}^{S} E^{q \frac{r+1}{r-1}}(t) d t+C_{3} E^{q+1}(T)+C_{4} E(T) .
$$

Recalling $q=\frac{r-1}{2}$, we get $q+1=q \frac{r+1}{r-1}=\frac{r+1}{2}$. Hence, the above inequality is rewritten as

$$
\begin{aligned}
\int_{T}^{S} E^{\frac{r+1}{2}}(t) d t & \leq 2 C_{3} E^{\frac{r+1}{2}}(T)+2 C_{4} E(T) \\
& \leq 2\left(C_{3} E^{\frac{r-1}{2}}(0)+C_{4}\right) E(T),
\end{aligned}
$$

where the last inequality follows from Remark 3.1. Finally, by letting $S \rightarrow+\infty$ in (31), (35) and using Lemma 3.3 with $G(t)=E(t)$, we complete the proof of Theorem 3.1.

Remark 3.3 According to the proof of Theorem 3.1, it is easy to see that the constants $\sigma, k_{1}$ and $k_{2}$ in Theorem 3.1 can be chosen as, respectively, $\sigma^{-1}=2 \max \left\{1, \frac{1}{a}\right\}+\frac{1}{2}\left(\frac{1}{L_{1}}+\frac{L_{2}}{a}\right)$, $k_{1}=e E(0)$, and $k_{2}=E(0)\left[2 \frac{r+1}{r-1}\left(C_{3} E^{\frac{r-1}{2}}(0)+C_{4}\right)\right]^{\frac{2}{r-1}}$ with $C_{3}=\frac{3 r-1}{r+1} \max \left\{1, \frac{1}{a}\right\}+\frac{a+L_{1} L_{2}}{a(r+1) L_{1}}, C_{4}=$ $\frac{1}{r+1}\left(\frac{1}{L_{1}}+\frac{L_{2}^{r}}{a}\right)\left[\left(1+\frac{1}{a}\right) \frac{r-1}{r+1}\right]^{\frac{r-1}{2}}$. This means that the coefficients of the exponential or polynomial decay rate are exactly determined only by the initial tension $a$, the initial energy $E(0)$ and the feedback control $u$. However, in the polynomial decay case, the order of decay rate is determined only by the feedback control $u$.

Finally, it is shown that the boundary control $u$ stabilizes the nonlinear Kirchhoff string. 
Theorem 3.2 Assume that assumption $(\mathrm{H})$ holds. Then there exist two constants $k_{1}^{\prime}, k_{2}^{\prime}>0$ such that for all $x \in(0,1)$ and $t \geq 0$,

$$
\begin{cases}|y(x, t)| \leq k_{1}^{\prime} e^{-\frac{\sigma t}{2}} & \text { if } r=1, \\ |y(x, t)| \leq k_{2}^{\prime} t^{-\frac{1}{r-1}} & \text { if } r>1\end{cases}
$$

where $k_{1}^{\prime}=\sqrt{\frac{2 k_{1}}{a}}, k_{2}^{\prime}=\sqrt{\frac{2 k_{2}}{a}}$ and $k_{1}, k_{2}, \sigma$ are given in Theorem 3.1.

Proof According to the fact that $y_{x}(0, t)=0$, for all $t \geq 0$, we get

$$
\begin{aligned}
|y(x, t)| & =\left|\int_{0}^{x} y_{x}(z, t) d z\right| \leq \int_{0}^{1}\left|y_{x}(z, t)\right| d z \\
& \leq\left(\int_{0}^{1} y_{x}^{2}(x, t) d x\right)^{\frac{1}{2}} \quad \text { (by the Cauchy inequality) } \\
& \leq \sqrt{\frac{2 E(t)}{a}} \quad \text { (by equation (14)) }
\end{aligned}
$$

for all $x \in(0,1)$ and $t \geq 0$. By combining (37) with (30) in Theorem 3.1, we complete the proof of Theorem 3.2.

\section{Numerical results}

In this section we consider a computational example for the closed-loop system (7a)-(7d). To illustrate the control performance of the boundary control law satisfying condition $(\mathrm{H})$, numerical simulations by using the finite element method (FEM) are performed. We use Lagrange 'hat' basis with FEM equidistant meshes. The system parameters used in the simulations are $a=2, b=3$. The initial conditions are $f(x)=0.6 \sin (5 x)$ and $g(x)=$ $0.5 \sin (3 x)$. That is we consider the following Kirchhoff system:

$$
\left\{\begin{array}{l}
y_{t t}(x, t)=\left[2+3 \int_{0}^{1} y_{x}^{2}(x, t) d x\right] y_{x x}(x, t), \\
y(0, t)=0 \\
{\left[a+b \int_{0}^{1} y_{x}^{2}(x, t) d x\right] y_{x}(1, t)=u\left(y_{t}(1, t)\right),} \\
y(x, 0)=0.6 \sin (5 x), \quad y_{t}(x, 0)=0.5 \sin (3 x) .
\end{array}\right.
$$

The dynamic responses of the controlled Kirchhoff string were simulated under two feedback control laws:

$$
u_{1}(x)=-2 x-\sin x, \quad x \in R
$$

and

$$
u_{2}(x)= \begin{cases}-\sqrt{|x|}, & |x|<1 \\ -x, & |x| \geq 1 .\end{cases}
$$

Obviously, the feedback control function $u_{1}$ satisfies the constraint condition $(\mathrm{H})$ with $r=1, L_{1}=1, L_{2}=3$, and $u_{2}$ satisfies the constraint condition $(\mathrm{H})$ with $r=2, L_{1}=L_{2}=1$. Then, according to Theorem 3.2, the asymptotic behavior of the transverse vibration of system (38) under control law $u_{1}$ (or control law $u_{2}$ ) possesses exponential decay (or poly- 

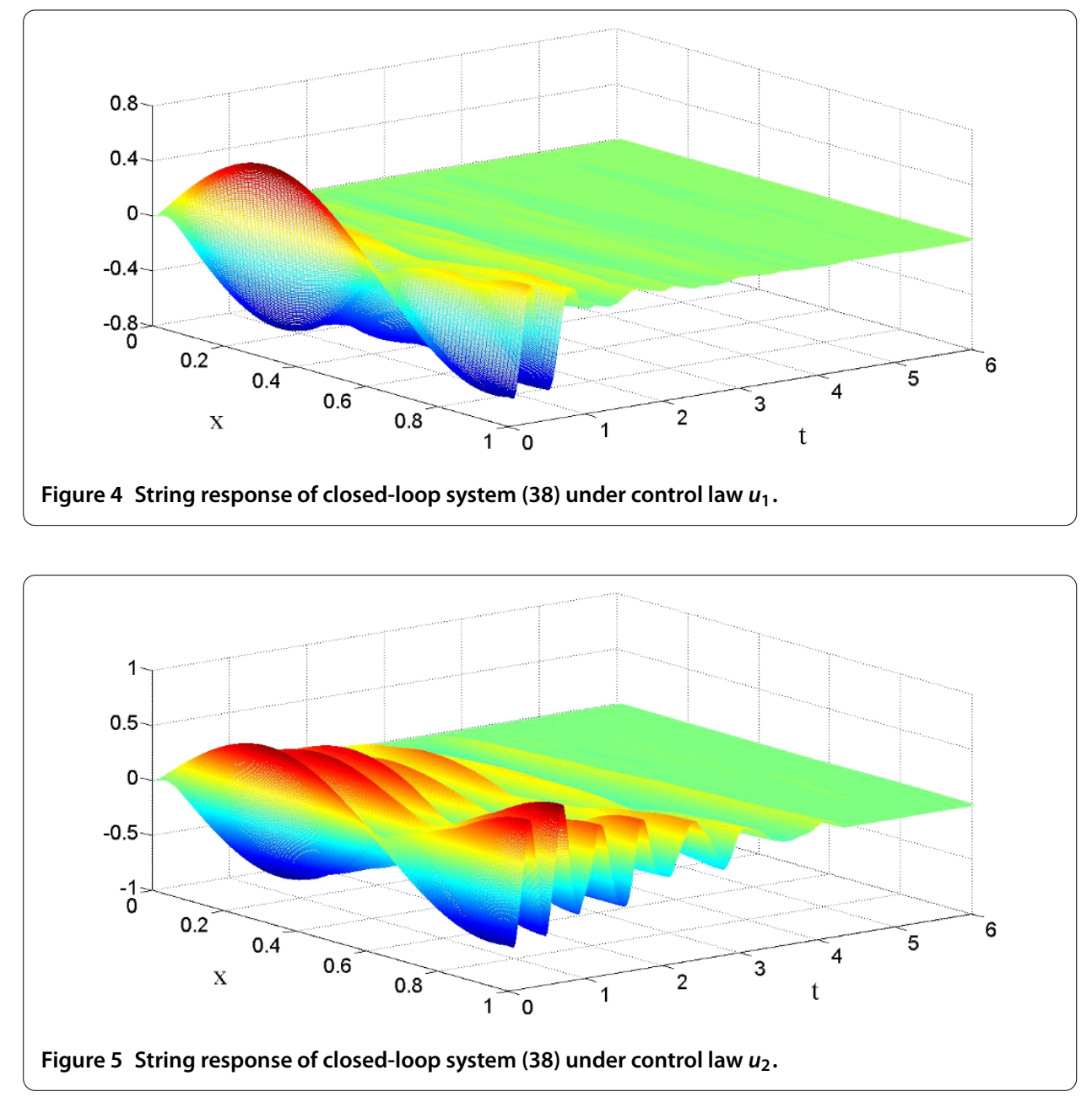

nomial decay with degree -1 , because $r=2$ ). The string response $y(x, t)$ of closed-loop system (38) with control law $u_{1}$ and control law $u_{2}$ are shown in Figure 4 and Figure 5, respectively. The corresponding transversal displacement at $x=0.5$ is shown in Figures 6 and 7, respectively.

From Figures 4 and 5 , it can bee seen that, in the case of control law $u_{2}$, the decay of the transverse vibration relatively slow compared to the case of control law $u_{1}$. Indeed, from Figure 6 and Figure 7, we know that in the case of control law $u_{1}$, the transverse vibration has been suppressed exponentially $\left(|y(0.5, t)| \leq K_{2} e^{-\sigma t}\right)$, whereas in the case of control law $u_{2}$, the transverse vibration has been suppressed polynomially $\left(|y(0.5, t)| \leq K_{3} t^{-1}\right)$. It is coincident with the results of Theorem 3.2.

\section{Appendix}

\section{A.1 Proof of Lemma 3.1}

Integrating by parts and (9) we get, for all $t \geq 0$,

$$
\begin{aligned}
\int_{0}^{1} y_{x}(x, t) y_{x t}(x, t) d x & =\int_{0}^{1}\left[y_{x}(x, t) y_{t}(x, t)\right]_{x} d x-\int_{0}^{1} y_{x x}(x, t) y_{t}(x, t) d x \\
& =y_{x}(1, t) y_{t}(1, t)-\int_{0}^{1} y_{x x}(x, t) y_{t}(x, t) d x .
\end{aligned}
$$




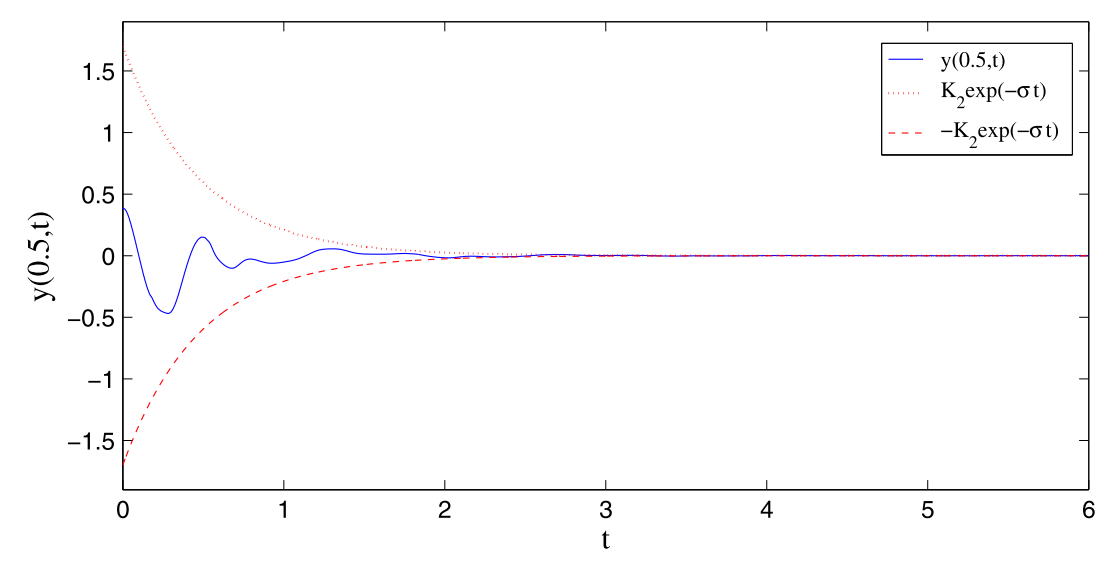

Figure 6 Transverse displacement of the string at $x=0.5$ under control law $u_{1}$.

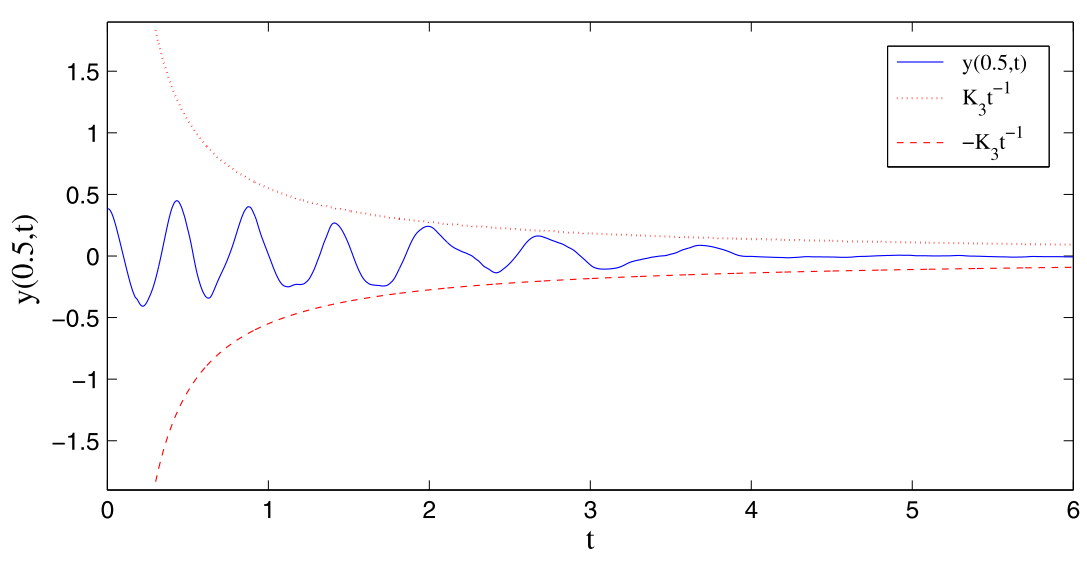

Figure 7 Transverse displacement of the string at $x=0.5$ under control law $u_{2}$.

So we obtain equation (11). Next, integrating by parts we compute

$$
\int_{0}^{1} x y_{x} y_{x x} d x=\frac{1}{2} \int_{0}^{1}\left[x y_{x}^{2}\right]_{x} d x-\frac{1}{2} \int_{0}^{1} y_{x}^{2} d x=\frac{1}{2} y_{x}^{2}(1, t)-\frac{1}{2} \int_{0}^{1} y_{x}^{2} d x
$$

for all $t \geq 0$. That is, equation (12) holds. Finally, we write

$$
\int_{0}^{1} x y_{x} y_{t t} d x=\int_{0}^{1}\left[x y_{x} y_{t}\right]_{t} d x-\int_{0}^{1} x y_{t} y_{x t} d x
$$

and

$$
\int_{0}^{1} x y_{t} y_{x t} d x=\frac{1}{2} \int_{0}^{1}\left[x y_{t}^{2}\right]_{x} d x-\frac{1}{2} \int_{0}^{1} y_{t}^{2} d x
$$

for all $t \geq 0$. Substituting equation (40) into equation (39), we obtain equation (13). Thus the proof of Lemma 3.1 is complete. 


\section{A.2 Proof of Claim 1}

Since $r>1$, hypothesis $(\mathrm{H})$ implies

$$
\begin{aligned}
& L_{1}|s|^{r} \leq|u(s)| \leq L_{2}|s|^{\frac{1}{r}} \quad \text { if }|s| \leq 1, \\
& L_{1}|s| \leq|u(s)| \leq L_{2}|s| \quad \text { if }|s|>1 .
\end{aligned}
$$

Hence, it is true that, for all $s \in R$,

$$
\begin{aligned}
& s^{2} \leq\left(-\frac{1}{L_{1}} u(s) s\right)^{\frac{2}{r+1}}-\frac{1}{L_{1}} u(s) s, \\
& u^{2}(s) \leq\left(-L_{2}^{r} u(s) s\right)^{\frac{2}{r+1}}-L_{2} u(s) s .
\end{aligned}
$$

Then, from (41) and Proposition 3.1 we have

$$
\begin{aligned}
& \int_{T}^{S} E^{q}(t) y_{t}^{2}(1, t) d t \\
& \quad \leq \int_{T}^{S} E^{q}(t)\left(-\frac{1}{L_{1}} u(t) y_{t}(1, t)\right)^{\frac{2}{r+1}} d t+\int_{T}^{S} E^{q}(t)\left(-\frac{1}{L_{1}} u(t) y_{t}(1, t)\right) d t \\
& \quad=\int_{T}^{S} E^{q}(t)\left(-\frac{E^{\prime}(t)}{L_{1}}\right)^{\frac{2}{r+1}} d t-\frac{1}{L_{1}} \int_{T}^{S} E^{q}(t) E^{\prime}(t) d t \\
& \quad \leq \int_{T}^{S} E^{q}(t)\left(-\frac{E^{\prime}(t)}{L_{1}}\right)^{\frac{2}{r+1}} d t+\frac{E^{q+1}(T)}{(q+1) L_{1}} .
\end{aligned}
$$

From Young's inequality, we have, for any $\alpha, \beta, \delta>0$,

$$
\alpha \beta \leq \delta^{\rho} \frac{\alpha^{\rho}}{\rho}+\delta^{-\rho^{\prime}} \frac{\beta^{\rho^{\prime}}}{\rho^{\prime}}
$$

where $\rho, \rho^{\prime}>1$ and $\frac{1}{\rho}+\frac{1}{\rho^{\prime}}=1$. Applying the above inequality with $\alpha=E^{q}(t), \beta=\left(-\frac{E^{\prime}(t)}{L_{1}}\right) \frac{2}{r+1}$, $\rho=\frac{r+1}{r-1}$ and $\rho^{\prime}=\frac{r+1}{2}$, we obtain, for any $\delta>0$,

$$
\begin{aligned}
& \int_{T}^{S} E(t)^{q}\left(-\frac{E^{\prime}(t)}{L_{1}}\right)^{\frac{2}{r+1}} d t \\
& \quad \leq \frac{r-1}{r+1} \delta^{\frac{r+1}{r-1}} \int_{T}^{S} E^{q \frac{r+1}{r-1}}(t) d t+\frac{2 \delta^{-\frac{r+1}{2}}}{(r+1) L_{1}} \int_{T}^{S}-E^{\prime}(t) d t \\
& \quad \leq \frac{r-1}{r+1} \delta^{\frac{r+1}{r-1}} \int_{T}^{S} E^{q \frac{r+1}{r-1}}(t) d t+\frac{2 \delta^{-\frac{r+1}{2}}}{(r+1) L_{1}} E(T) .
\end{aligned}
$$

By inserting (45) into (43), we deduce inequality (32) in Claim 1. Similarly, from (42) and Proposition 3.1 we have

$$
\begin{aligned}
& \int_{T}^{S} E^{q}(t) u^{2}\left(y_{t}(1, t)\right) d t \\
& \quad \leq \int_{T}^{S} E^{q}(t)\left(-L_{2}^{r} u(t) y_{t}(1, t)\right)^{\frac{2}{r+1}} d t+L_{2} \int_{T}^{S} E^{q}(t)\left(-u(t) y_{t}(1, t)\right) d t
\end{aligned}
$$




$$
\begin{aligned}
& =\int_{T}^{S} E^{q}(t)\left(-L_{2}^{r} E^{\prime}(t)\right)^{\frac{2}{r+1}} d t-L_{2} \int_{T}^{S} E^{q}(t) E^{\prime}(t) d t \\
& \leq \int_{T}^{S} E^{q}(t)\left(-L_{2}^{r} E^{\prime}(t)\right)^{\frac{2}{r+1}} d t+\frac{L_{2}}{(q+1)} E^{q+1}(T) .
\end{aligned}
$$

Applying again inequality (44) with $\alpha=E^{q}(t), \beta=\left[-L_{2}^{r} E^{\prime}(t)\right] \frac{2}{r+1}, \rho=\frac{r+1}{r-1}$ and $\rho^{\prime}=\frac{r+1}{2}$, we obtain, for any $\delta>0$

$$
\begin{aligned}
& \int_{T}^{S} E(t)^{q}\left(-L_{2}^{r} E^{\prime}(t)\right)^{\frac{2}{r+1}} d t \\
& \quad \leq \frac{r-1}{r+1} \delta^{\frac{r+1}{r-1}} \int_{T}^{S} E^{q^{\frac{r+1}{r-1}}}(t) d t+\frac{2 L_{2}^{r} \delta^{-\frac{r+1}{2}}}{(r+1)} \int_{T}^{S}-E^{\prime}(t) d t \\
& \quad \leq \frac{r-1}{r+1} \delta^{\frac{r+1}{r-1}} \int_{T}^{S} E^{q^{r+1}}(t) d t+\frac{2 L_{2}^{r} \delta^{\frac{r+1}{2}}}{(r+1)} E(T) .
\end{aligned}
$$

Inserting (47) into (46), we get inequality (33) in Claim 1.

\section{Competing interests}

The authors declare that they have no competing interests.

\section{Authors' contributions}

All authors contributed equally to the manuscript and read and approved the final manuscript.

\section{Author details}

${ }^{1}$ Department of Mathematics, Harbin University of Science and Technology, Harbin, 150080, P.R. China. ${ }^{2}$ Department of Electronics Science and Technology, Harbin University of Science and Technology, Harbin, 150080, P.R. China.

\section{Acknowledgements}

This work is supported by the National Natural Science Foundation of China (Grant 11226128) and the Natural Science Foundation of Heilongjiang Province of China (Grant F201113).

Received: 22 April 2013 Accepted: 6 August 2013 Published: 21 October 2013

\section{References}

1. Chen, G, Delfour, MC, Krall, AM, Payre, G: Modeling, stabilization and control of serially connected beams. SIAM J. Control Optim. 25, 526-546 (1987)

2. Lagnese, J: Decay of solutions of wave equations in a bounded domain with boundary dissipation. J. Differ. Equ. 50, 163-182 (1983)

3. Shahruz, SM: Bounded-input bounded-output stability of a damped nonlinear string. IEEE Trans. Autom. Control 41, 1179-1182 (1996)

4. Guo, BZ: Riesz basis approach to the stabilization of a flexible beam with a tip mass. SIAM J. Control Optim. 39 , 1736-1747 (2001)

5. Fung, RF, Wu, JW, Wu, SL: Exponential stabilization of an axially moving string by linear boundary feedback. Automatica 35, 177-181 (1999)

6. Morgül, Ö: Stabilization disturbance rejection for the beam equation. IEEE Trans. Autom. Control 46(12), 1913-1918 (2001)

7. Smyshlyaev, A, Guo, B-Z, Krstic, M: Arbitrary decay rate for Euler-Bernoulli beam by backstepping boundary feedback IEEE Trans. Autom. Control 54(5), 1134-1140 (2009)

8. Wang, JJ, Li, QH: Active vibration control methods of axially moving materials - a review. J. Vib. Control 10,475-491 (2004)

9. Luo, ZH, Guo, BZ, Morgül, Ö: Stability and Stabilization of Infinite Dimensional Systems with Applications. Communications and Control Engineering Series. Springer, London (1999)

10. Kirchhoff, G: Vorlesungen über Mechanik. Teubner, Stuttgart (1883)

11. Arosio, A, Panizzi, S: On the well-posedness of the Kirchhoff string. Trans. Am. Math. Soc. 348(1), 305-330 (1996)

12. Ono, K: Global existence, decay, and blow up of solutions for some mildly degenerate nonlinear Kirchhoff strings. J. Differ. Equ. 137, 273-301 (1997)

13. Shahruz, SM, Krishna, LG: Boundary control of a non-linear string. J. Sound Vib. 195, 169-174 (1996)

14. Oplinger, DW: Frequency response of a nonlinear stretched string. J. Acoust. Soc. Am. 32, 1529-1538 (1960)

15. $\mathrm{Wu}, \mathrm{YH}, \mathrm{Xue}, \mathrm{XP}$, Shen, TL: Absolute stability of the Kirchhoff string with sector boundary control (under review)

16. Chen, G: Energy decay estimates and exact boundary value controllability for the wave equation in a bounded domain. J. Math. Pures Appl. 58, 249-273 (1979)

17. Chen, G: A note on boundary stabilization of the wave equation. SIAM J. Control Optim. 19, 106-113 (1981) 
18. Lagnese, JE: Note on boundary stabilization of wave equations. SIAM J. Control Optim. 26, 1250-1256 (1988)

19. Shahruz, MS: Suppression of vibration in a nonlinear axially moving string by boundary control. ASME Des. Eng. Tech Conf. 106(1), 6-14 (1997)

20. Li, TC, Hou, ZC: Exponential stabilization of an axially moving string with geometrical nonlinearity by linear boundary feedback. J. Sound Vib. 296, 861-870 (2006)

21. Kobayashi, T: Boundary position feedback control of Kirchhoff's non-linear strings. Math. Methods Appl. Sci. 27, 79-89 (2004)

22. Komornik, V: Exact Controllability and Stabilization. RAM: Research in Applied Mathematics. Masson, Paris; Wiley, Chichester (1994)

doi:10.1186/1687-2770-2013-215

Cite this article as: Wu and Wang: Exponential and polynomial stabilization of the Kirchhoff string by nonlinear boundary control. Boundary Value Problems 2013 2013:215.

Submit your manuscript to a SpringerOpen ${ }^{\odot}$ journal and benefit from:

- Convenient online submission

- Rigorous peer review

- Immediate publication on acceptance

- Open access: articles freely available online

- High visibility within the field

- Retaining the copyright to your article 